\title{
A small jet in Chamaeleon I powered by a low-luminosity source ${ }^{\star}$
}

\author{
F. Comerón ${ }^{1}$ and B. Reipurth ${ }^{2}$ \\ ${ }^{1}$ European Southern Observatory, Karl-Schwarzschild-Strasse 2, 85748 Garching bei München, Germany \\ e-mail: fcomeron@eso.org \\ 2 Institute for Astronomy, University of Hawaii, 640 N. Aohoku Place, Hilo, HI 96720, USA \\ e-mail: reipurth@ifa.hawaii.edu
}

Received 1 August 2006 / Accepted 6 September 2006

\section{ABSTRACT}

\begin{abstract}
We report the discovery of a small bipolar Herbig-Haro jet, $\mathrm{HH} 872$, powered by the low-luminosity source ESO-H $\alpha$ 574 in Chamaeleon I. The end-to-end projected size of the jet in [SII] images is only 3150 AU. Infrared images marginally resolve the central source showing a structure elongated perpendicularly to the jet axis, possibly a disk seen at a large angle with respect to the plane of the sky. The brightest feature of the jet, peaking at a distance of 1 " 6 from the central source, is likely to result from enhanced outflow activity in the last decade.
\end{abstract}

Key words. stars: low-mass, brown dwarfs - stars: pre-main sequence - ISM: jets and outflows

\section{Introduction}

Increasing evidence suggests that the signposts of accretion that characterize the $\mathrm{T}$ Tauri phase among solar-type stars extend well into the substellar realm (see e.g. Luhman et al. 2006 for a recent review) and even into the giant-planetmass domain (Muzerolle et al. 2005; Mohanty et al. 2005), bearing important constraints on the properties of their circum(sub)stellar disks (Alexander \& Armitage 2006). Recent observations have also revealed outflow signatures around very low-mass stars and brown dwarfs (Fernández \& Comerón 2001, 2005; Barrado y Navascués et al. 2004; Luhman 2004b; Whelan et al. 2005, 2006; Scholz \& Jayawardhana 2006).

Recent surveys covering virtually the entire Chamaeleon I star-forming clouds (Comerón et al. 2004; Luhman 2004a; López Martí et al. 2004) have revealed many new very low-mass stars and brown dwarfs. Among them, Comerón et al. (2004) reported the existence of a very faint source, ESO-H $\alpha 574$, with the $V R_{\mathrm{C}} I_{\mathrm{C}} J H K_{\mathrm{S}}$ colors of lightly reddened late-type stars and a rich emission-line spectrum dominated by the forbidden lines associated with outflows. Lines normally used as accretion diagnostics, such as HeI and CaII, are by comparison weak or absent. Comerón et al. (2004) proposed that ESO-H $\alpha 574$ may be a lowmass star with a strongly-veiled spectrum seen in scattered light due to an edge-on disk blocking our direct view of the photosphere of the central object. This may explain its low luminosity $\left(R_{\mathrm{C}}=17.87\right)$, which taken at face value would place the object below the main sequence. In this paper we report the discovery of a well-developed jet, here designated $\mathrm{HH} 872$, detected in [SII] imaging of ESO-H $\alpha 574$, whose further study can yield important clues to the validity of this scenario.

* Based on observations obtained in Service Mode at the European Southern Observatory using the Very Large Telescope on Cerro Paranal (observing runs 075.C-0809(A) and (B)).

\section{Observations}

Our [SII] observations were carried out in Service Mode using the visible imager and low resolution spectrograph FORS1 at the ESO Very Large Telescope (VLT) on the night of 21 July 2005. The $K_{\mathrm{S}}$-band imaging was obtained with ISAAC, the infrared camera and low-resolution spectrograph at the VLT, also in Service Mode on the night of 26 March 2005. The field around ESO-H $\alpha 574\left(\alpha_{2000}=11^{\mathrm{h}} 16^{\mathrm{m}} 03^{\mathrm{s}} 7, \delta_{2000}=-76^{\circ} 24^{\prime} 53^{\prime \prime}\right)$ was observed with a [SII] filter, centered on $6728 \AA$ and with a fullwidth at half maximum $(F W H M)$ of $66 \AA$. The filter response is approximately uniform within an interval only slightly narrower than the $F W H M$ of the filter. Two exposures of 15 min each were obtained under good seeing conditions yielding a quality of $0^{\prime \prime} 70$ on the combined image. The ISAAC $K_{\mathrm{S}}$ observation was a set of $5 \times 45 \mathrm{~s}$ exposures with small telescope offsets of $15^{\prime \prime}$ amplitude in between. We used the pipeline-reduced images of both observations in the data set delivered to us (Silva \& Péron 2004).

An approximate calibration of the [SII] flux was carried out taking the existing $R_{\mathrm{C}}$-band images of the field (Comerón et al. 2004) as a reference. The $R_{\mathrm{C}}$ magnitudes of the stars in that field were converted to flux per unit wavelength $f_{R_{\mathrm{C}}}$, using $f_{R_{\mathrm{C}}}=1.80 \times 10^{-5-0.4 R_{\mathrm{C}}} \mathrm{erg} \mathrm{cm}^{-2} \mathrm{~s}^{-1} \mu \mathrm{m}^{-1}$ (Bessell 1979) and assuming that $f_{R_{\mathrm{C}}} \simeq f_{[\mathrm{SII}]}$ for those stars, given the proximity in central wavelengths of the $R_{\mathrm{C}}$ and [SII] filters. The total flux $F_{[\mathrm{SII}]}$ within the window defined by the [SII] transmission curve was then approximated by $F_{[\mathrm{SII}]}=f_{[\mathrm{SII}]} \Delta \lambda$, where $\Delta \lambda$ is the $F W H M$ of the [SII] filter. Dividing the $F_{[\mathrm{SII}]}$ thus derived for each star by the analog-to-digital units (ADU) recorded in the detector allowed us to obtain the conversion factor needed to transform ADUs into power received from the source per unit surface. Since we lack images sampling the continuum adjacent to the [SII] lines, we cannot separate the contribution to the flux received within the [SII] filter window from the stellar photosphere and from the line emission at the position of the central source of ESO-H $\alpha$ 574. Outside the central source we assume 


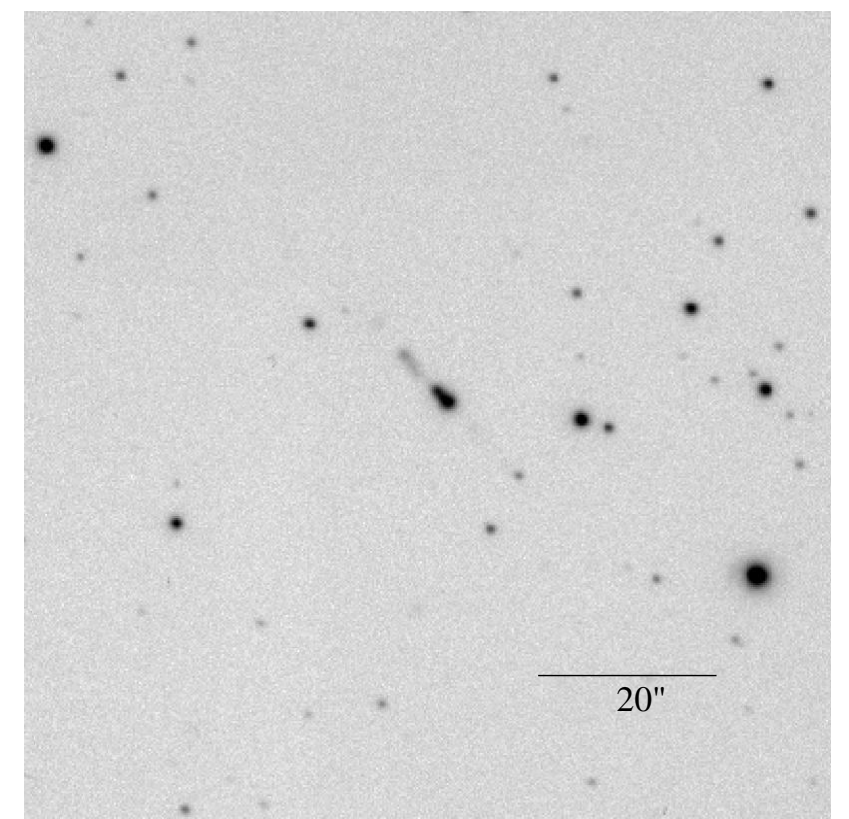

Fig. 1. Image of the field around ESO-H $\alpha 574$ through the [SII] filter, combining two exposures of 15 min obtained under 0 " 70 seeing. North is at the top and east to the left.

that the $[\mathrm{SII}]$ emission is much more intense than any possible contribution to the continuum, such as possible scattered light from the source.

\section{Results}

The [SII] image of ESO-H $\alpha 574$ (Fig. 1) shows a well-developed jet, $\mathrm{HH} 872$, extending towards the northeast that can be traced out to a distance of $11^{\prime \prime} 3$ from the central source. A much fainter jet can be seen towards the southwest reaching out to a distance of about $8^{\prime \prime} 3$. The northeastern jet is composed of four distinct knots, as indicated in Fig. 2: the brightest one, knot A, stretches $1^{\prime \prime} 6$ from the star, with a peak surface brightness of $8.7 \times 10^{-15} \mathrm{erg} \mathrm{cm}^{-2} \mathrm{~s}^{-1} \operatorname{arcsec}^{-2}$. It is highly reminiscent of $\mathrm{HH} 600$, a knot in $\mathrm{H} \alpha$ and [SII] images of Par-Lup3-4 reported by Fernández \& Comerón (2005). A second component, knots $\mathrm{B}$ and $\mathrm{C}$, is $\sim 3^{\prime \prime}$ away, knot $\mathrm{C}$ being the brightest with a surface brightness of $5.7 \times 10^{-16} \mathrm{erg} \mathrm{cm}^{-2} \mathrm{~s}^{-1} \operatorname{arcsec}^{-2}$. The jet ends in the distinct, faint knot $\mathrm{D}$ separated from the knot $\mathrm{C}$ by a gap of another $3^{\prime \prime}$. The structure in the fainter southwestern jet is less obvious, with a peak surface brightness $1.0 \times 10^{-16} \mathrm{erg} \mathrm{cm}^{-2} \mathrm{~s}^{-1} \operatorname{arcsec}^{-2}$ in knot E. The total flux of $\mathrm{HH} 872$ is estimated as $F_{[\mathrm{SII}]}=9.8 \times 10^{-15} \mathrm{erg} \mathrm{cm}^{-2} \mathrm{~s}^{-1}$, excluding the flux unresolved from the central source, which as explained in Sect. 2 cannot be disentangled from the photospheric continuum. At the $160 \mathrm{pc}$ distance of the Chamaeleon I cloud (Wichmann et al. 1998), the luminosity in the [SII] lines is $L_{[\mathrm{SII}]}=7.5 \times 10^{-6} L_{\odot}$, and the projected end-to-end length of $\mathrm{HH} 872$ is $3140 \mathrm{AU}(0.015 \mathrm{pc})$.

No trace of $\mathrm{HH} 872$ is seen in the $K_{\mathrm{S}}$ image (Fig. 3). However, the image quality ( $0^{\prime \prime} 78$ in the final combined image) allows us to marginally resolve the central source, which appears slightly elongated in the northwest-southeast direction, roughly perpendicular to the jet. The estimated size of the source, obtained from the quadratic difference between the major and minor axes of the image of the source, respectively, and the FWHM of the images of unresolved sources in the field, is $0^{\prime \prime} 41 \times 0^{\prime \prime} 26$ $(66 \times 42 \mathrm{AU})$.

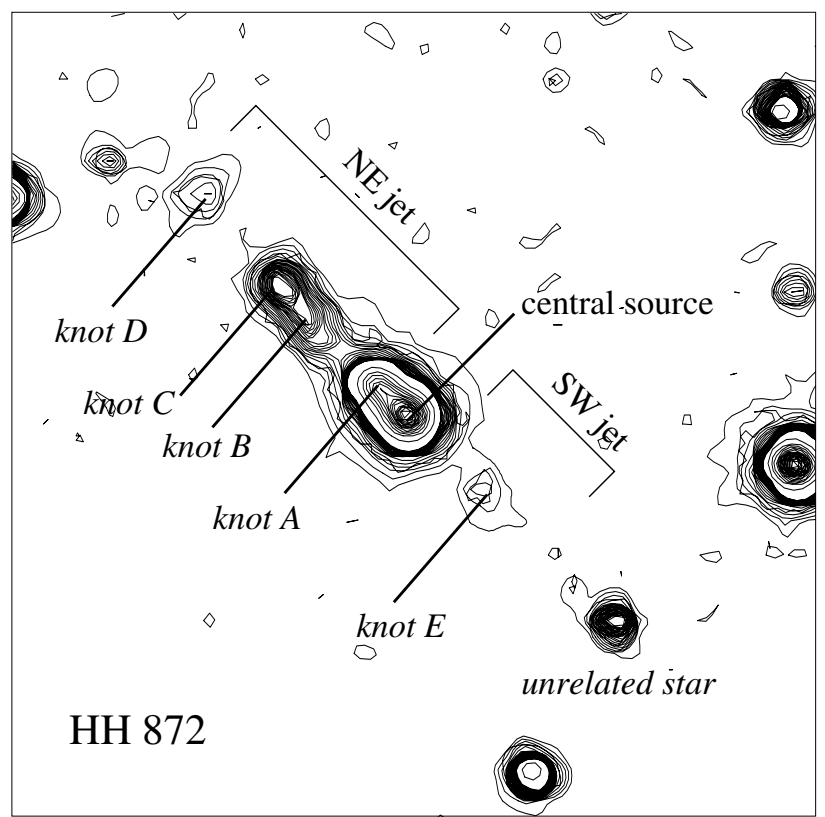

Fig. 2. Contour plot of the central $30^{\prime \prime} \times 30^{\prime \prime}$ area of Fig. 1 showing details of the structure of the HH 872 jet, particularly the existence of three distinct components of emission in the northeastern jet. The contours corresponding to the higher intensities are separated by larger flux intervals in order to more clearly show the structure of the base of the northeastern jet (knot A) in the vicinity of the central star.

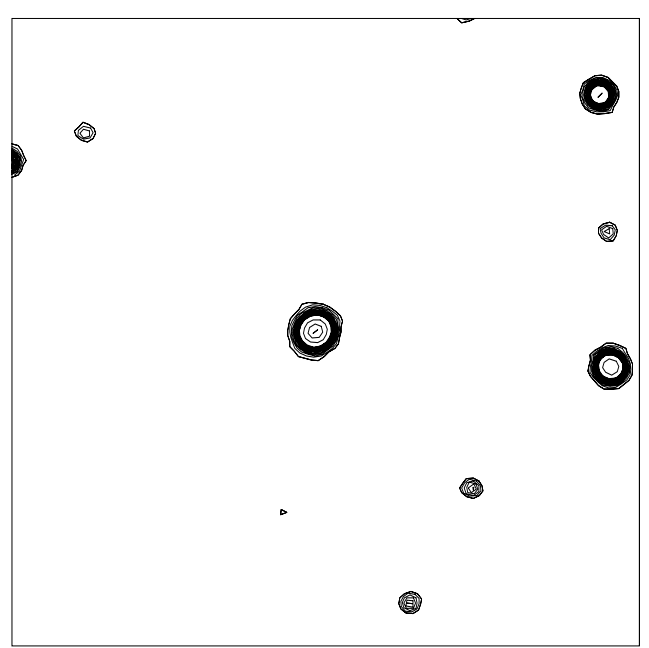

Fig. 3. Contour plot of the $K_{\mathrm{S}}$ image of the area approximately coinciding with the one presented in Fig. 2. Hints of an elongation of ESO-H $\alpha 574$ in the northwest-southeast direction can be seen. The analysis of the point-spread function of the stars in the field shows that they are all round with a full-width at half-maximum of $0^{\prime \prime} 78 \pm 0$ " 02 and that ESO-H $\alpha 574$ is slightly extended and elongated, with major and minor axes of $0^{\prime \prime} 88$ and $0^{\prime \prime} 82$, respectively.

The $V R_{\mathrm{C}} I_{\mathrm{C}} J H K_{\mathrm{S}}$ photometry of ESO-H $\alpha 574$ is presented in Comerón et al. (2004). Unfortunately, its location in the external parts of the Chamaeleon I cloud has excluded it from observations of Chamaeleon I currently being carried out by the Spitzer Space Telescope. There is, however, a tentative IRAS detection at $100 \mu \mathrm{m}$ near its position, which may be due to cold circumstellar material. 


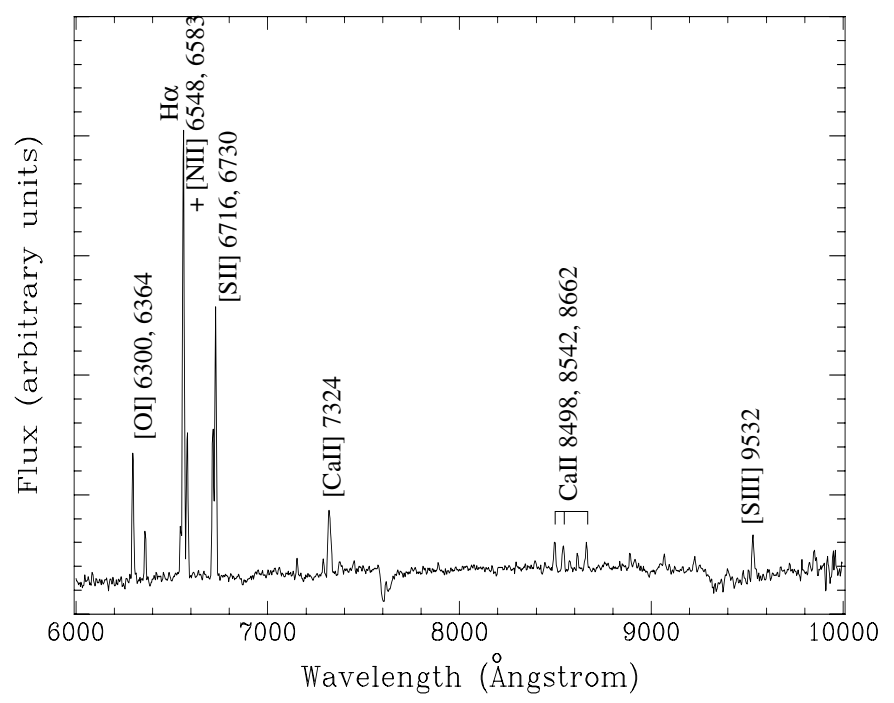

Fig. 4. Spectrum of ESO-H $\alpha$ 574, adapted from Comerón et al. (2004). The most prominent lines are indicated. The full list of emission lines detected in the spectrum can be found in that paper and includes numerous forbidden transitions in addition to the intense ones labeled here. No absorption features other than telluric bands are noticeable.

\section{Discussion}

\subsection{Physical properties}

We can crudely estimate some physical characteristics of $\mathrm{HH} 872$ by using line ratios from the spectrum of ESO-H $\alpha 574$ presented by Comerón et al. (2004) and by assuming them to be representative of the entire jet. The spectrum (Fig. 4) samples only the base of the jet, where other contributions to $\mathrm{H} \alpha$ may be expected, most notably accretion. Therefore, both the $[\mathrm{SII}](6716+6731) / \mathrm{H} \alpha$ and the $[\mathrm{NII}](6548+6543) / \mathrm{H} \alpha$ ratios are expected to be lower limits to the ratios encountered along the jet. With this caveat, we have applied the analysis of Bacciotti et al. (1995). Assuming solar abundances, we obtain an electron temperature $T_{\mathrm{e}}=6200 \mathrm{~K}$ and a hydrogen ionization fraction $x=0.25$. The electron density, from the [SII](6716)/[SII](6731) ratio, is $n_{\mathrm{e}} \simeq 3000 \mathrm{~cm}^{-3}$ (Osterbrock 1989). Assuming that the measured $[\mathrm{SII}](6716+6731) / \mathrm{H} \alpha$ ratio is approximately the same as the ratios between the [SII] and $\mathrm{H} \alpha$ luminosities over the entire jet, we obtain $L_{\mathrm{H} \alpha}=8.8 \times$ $10^{-6} L_{\odot}$. Taking into account that these are conditions at the base of the jet and that we have most likely underestimated [NII]/H $\alpha$ and [SII] $/ \mathrm{H} \alpha$, the average $x$ and $L_{\mathrm{H} \alpha}$ in the jet are likely to be lower, and $T_{\mathrm{e}}$ higher. Nevertheless, the limits found on $n_{\mathrm{e}}, x$, and $T_{\mathrm{e}}$ are similar to the values determined in other, much betterstudied T Tauri jets (e.g., Podio et al. 2006).

Estimates of the expected $\mathrm{H} \alpha$ luminosity $L_{\mathrm{H} \alpha}$ of the shockbounded internal working surfaces of brown dwarf-driven jets have been provided by Masciadri \& Raga (2004). Assuming that the approximate proportionality between mass loss and accretion rate in T Tauri stars (Hartigan et al. 1995) holds for brown dwarfs, and considering the rough proportionality between the accretion rate and the square of the mass of the central object (Muzerolle et al. 2005), the relationship between $L_{\mathrm{H} \alpha}$ and the mass-loss rate in the jet suggests that jets powered by brown dwarfs must be typically two orders of magnitude fainter than those powered by solar-type $\mathrm{T}$ Tauri stars. In the case of the $\mathrm{HH} 872$, the scenario worked out by Masciadri \& Raga (2004) is most likely found in knot A, where the freshly ejected gas from the star first encounters slower moving gas ejected earlier. Our estimate of $L_{\mathrm{H} \alpha} \simeq 6.7 \times 10^{-6} L_{\odot}$ for this segment is in principle in better agreement with Masciadri \& Raga's prediction for a brown dwarf-powered jet than for a classical $\mathrm{T}$ Tauri-driven jet. We must nevertheless note the large scatter in the accretion rate vs. central mass relationship (Luhman et al. 2006), consequently resulting in a large scatter in $L_{\mathrm{H} \alpha}$ at a given central mass stemming from this reason alone. Furthermore, $L_{\mathrm{H} \alpha}$ scales with the third power of the shock velocity, which is in turn related to the time variability of the jet, making $L_{\mathrm{H} \alpha}$ very sensitive to it. Finally, as noted above, our own estimate of $L_{\mathrm{H} \alpha}$ is indirect and considerably uncertain. Therefore, although the inferred $L_{\mathrm{H} \alpha}$ hints at ESO $\mathrm{H} \alpha 574$ being a very low-mass star or brown dwarf, model predictions are far too uncertain, given the lack of direct knowledge of the quantities involved, to rule out a more massive central source.

\subsection{Size and timescales}

The 11"3 angular extent of the northeastern jet corresponds to a projected $0.009 \mathrm{pc}$ at the distance of $160 \mathrm{pc}$. The jet ejection velocity is unknown but is expected to be of the order of the escape velocity of the central object, which for young objects is little sensitive to their mass since $M / R$ varies little over the range from solar-type stars to low-mass brown dwarfs. Assuming a projected jet velocity of $100 \mathrm{~km} \mathrm{~s}^{-1}$ the dynamical timescale is of order of 100 years, and the brightest segment at the base of the northeastern jet may thus result from a mass loss episode having started only about a decade ago. With 4 knots in the northeastern jet, the mean mass-loss variability timescale is $\sim 25$ years. However, given the large variations found among individual jets (e.g. velocities in the $300-600 \mathrm{~km} \mathrm{~s}^{-1}$ range are observed in the HH 111 jet; Reipurth et al. 1997), these quantities are just indicative.

A high-sensitivity survey of most of the Chamaeleon I cloud searching for [SII] emission has been recently presented by Wang \& Henning (2006), who report 14 new Herbig-Haro objects and detailed observations of the previously known $\mathrm{HH} 48$ to HH 50. Three of the new objects have a jet morphology, with the others appearing as knots or patches. ESO-H $\alpha$ 574, located in the northeastern outskirts of the Chamaeleon I North cloud, was slightly outside their surveyed area. The peak surface brightness of $\mathrm{HH} 872,8.8 \times 10^{-15} \mathrm{erg} \mathrm{cm}^{-2} \mathrm{~s}^{-1} \operatorname{arcsec}^{-2}$, is similar to the typical brightness of the objects observed by Wang \& Henning (2006). However, none of the [SII]-emitting features reported by Wang \& Henning displays the compact jet structure and the unambiguous association with its central source of HH 872. Moreover, the distances to their exciting sources, in the cases where the latter can be identified, are always much larger than the size of $\mathrm{HH} 872$ jet, including the three instances (HH 51, $\mathrm{HH}$ 909, and HH 908-HH-910) in which the suspected exciting sources are either very low-mass stars or brown dwarfs.

\section{Conclusions}

The discovery of $\mathrm{HH} 872$, an optical jet powered by ESO-H $\alpha$ 574, is perhaps not surprising given the previous detection of strong outflow signatures in the spectrum of the central source (Comerón et al. 2004). The limited information available thus far allows us to obtain crude estimates of the electron density, temperature, and ionization fraction in the jet, which are similar to those of jets excited by $\mathrm{T}$ Tauri stars. The morphology of the central source, which is barely resolved in the 
$K_{\mathrm{S}}$ band, indicates a flattened structure elongated in the direction perpendicular to the jet, suggesting a disk viewed at a large angle from the pole-on direction. In this regard ESO-H $\alpha 574$ may be similar to $\mathrm{LkH} \alpha 263 \mathrm{C}$, a young stellar object discovered by Jayawardhana et al. (2002) in MBM 12 with an estimated spectral type of M0 and a well-resolved nearly edge-on disk. The spectrum of $\mathrm{LkH} \alpha 263 \mathrm{C}$ also displays emission lines characteristic of outflows, although with a lower intensity that suggests that its possible jet, as yet undetected, should be much less prominent than the one reported here. On the other hand, our observations rule out an edge-on disk as extended as that around $\mathrm{LkH} \alpha 263 \mathrm{C}$, as the estimated physical size of the central source is roughly five times smaller than that reported by Jayawardhana et al. (2002).

The properties of the jet do not provide a definitive answer as to the nature of the central source. In particular, the question of whether or not the faintness of ESO-H $\alpha 574$ may actually be due to blocking by a circumstellar disk remains open. Nevertheless, both the low luminosity estimated for the internal working surface closest to the star and the small overall size of the structure hint at a very low mass of the central source. Assuming a jetejection velocity of $\sim 100 \mathrm{~km} \mathrm{~s}^{-1}$, the length scale of the features in $\mathrm{HH} 872$ implies the record of the mass ejection history of the source during the past century, and the brightest part of the jet, reaching only $1^{\prime \prime} 6$ from the central object, may be the result of a mass loss outburst having developed over the past decade. More observations, in particular high-resolution spectroscopy along the jet, yielding accurate line ratios and kinematics, and monitoring of the evolution of $\mathrm{HH} 872$ over a timescale of a few years, will be needed to better characterize the properties of the jet and to constrain those of the central source. However, the tentative conclusions presented here already suggest that ESO-H $\alpha 574$ is a good candidate for being one of the lowest mass sources known to power a Herbig-Haro outflow, opening the door to the detailed observational study of jets near the end of the stellar mass spectrum or possibly beyond.
Acknowledgements. We thank the ESO staff on Cerro Paranal who carried out the Service Mode observations, the ESO User Support Department for their valuable assistance in the preparation of our observations, and the ESO Data Flow Operations Group for the preparation of our data package. We also appreciate the comments of the referee, Ray Jayawardhana, pointing out the possible similarity between $\mathrm{ESO}-\mathrm{H} \alpha 574$ and $\mathrm{LkH} \alpha 263 \mathrm{C}$.

\section{References}

Alexander, R. D., \& Armitage, P. J. 2006, ApJ, 639, L83

Bacciotti, F., Chiuderi, C., \& Oliva, E. 1995, A\&A, 296, 185

Barrado y Navascués, D., Mohanty, S., \& Jayawardhana, R. 2004, ApJ, 604, 284 Bessell, M. S. 1979, PASP, 91, 589

Comerón, F., Reipurth, B., Henry, A., \& Fernández, M. 2004, A\&A, 417, 583

Fernández, M., \& Comerón, F. 2001, A\&A, 380, 264

Fernández, M., \& Comerón, F. 2005, A\&A, 440, 1119

Hartigan, P., Edwards, S., \& Ghandour, L. 1995, ApJ, 452, 736

Jayawardhana, R., Luhman, K. L., D’Alessio, P., \& Stauffer, J. R. 2002, ApJ, 571, L51

López Martí, B., Eislöffel, J., Scholz, A., \& Mundt, R. 2004, A\&A, 416, 555

Luhman, K. L. 2004a, ApJ, 602, 816

Luhman, K. L. 2004b, ApJ, 617, 1216

Luhman, K. L., Joergens, V., Lada, C., et al. 2006, in Protostars and Planets V, ed. B. Reipurth, D. Jewitt, \& K. Keil (Univ. of Arizona Press)

Masciadri, E., \& Raga, A. 2004, ApJ, 615, 850

Mohanty, S., Jayawardhana, R., \& Basri, G. 2005, ApJ, 626, 498

Muzerolle, J., Luhman, K. L., Briceño, C., Hartmann, L., \& Calvet, N. 2005, ApJ, 625, 906

Osterbrock, D. E. 1989, Astrophysics of Gaseous Nebulae and Active Galactic Nuclei (University Science Books)

Podio, L., Bacciotti, F., Nisini, B., et al. 2006, A\&A, in press

Reipurth, B., Hartigan, P., Heathcote, S., Morse, J. A., \& Bally, J. 1997, AJ, 114, 757

Scholz, A., \& Jayawardhana, R. 2006, ApJ, 638, 1056

Silva, D. R., \& Péron, M. 2004, The Messenger, 118, 2

Wang, H., \& Henning, T. 2006, ApJ, in press

Whelan, E. T., Ray, T. P., Bacciotti, F., et al. 2005, Nature, 435, 652

Whelan, E. T., Ray, T. P., Bacciotti, F., \& Jayawardhana, R. 2006, New Astron. Rev., 49, 582

Wichmann, R., Bastian, U., Krautter, J., Jankovics, I., \& Ruciński, S. M. 1998, MNRAS, 301, L39 